\title{
Reliability Measures of a Computer System with Priority for Repair and Hardware Redundancy
}

\author{
V.J. Munday \\ M.D. University, Rohtak-124001 (India) \\ Department of Statistics,
}

\author{
S.C. Malik \\ Department of Statistics, \\ M.D. University, Rohtak-124001 (India)
}

\begin{abstract}
This paper concentrates on the evaluation of reliability measures of a computer system by introducing the concept of priority of hardware repair over software up-gradation. The system operates with one more hardware in cold standby. The failure times of hardware and software are independent random variables which follow negative exponential distribution. The repair facility (called server) attends the faults immediately which occur during operation of the system. Repair of the hardware is done at failure while software undergoes for up-gradation. The distributions of hardware repair and software up-gradation times are taken as arbitrary with different probability density functions. The system model has been analyzed using semi-Markov process and regenerative point technique. The trends of some important measures of system effectiveness have been observed for arbitrary values of the parameters. The profit of the present model has also been compared with that of the system model in which no priority is given to hardware repair.
\end{abstract}

\section{Keywords}

Computer System, Hardware Redundancy, Priority to Repair, Software Up-gradation, Hardware Repair and Reliability Measures

\section{INTRODUCTION}

The rigorous reliability requirements of computer systems have forced the hardware and software engineers to probe the techniques that can be used in improving their performance. The technique of redundancy in different standby modes has been proved as one of the effective way to meet out this requirement. Therefore, over the few years, a lot of research work on stochastic modeling of repairable and non repairable systems has been appeared in the literature of reliability with the technique of redundancy. Welke et al. (1995) have discussed reliability modeling of a hardware/software system. The technique of unit wise redundancy in cold standby mode has also been used in computer systems. Malik and Anand (2010), Kumar et al. (2013) and Malik (2013) analyzed different computer system models with unit wise cold standby redundancy and different repair policies. But, it is also proved that component wise redundancy is better than unit wise redundancy so far as reliability is concerned. Malik and Munday (2014) developed a stochastic model for a computer system with hardware component in cold standby redundancy. Further, reliability of operating systems may be improved by giving priority in repair disciplines of one unit over the other. Anand and Malik (2012) studied a cold standby computer system by giving priority to hardware repair activities over software replacement.

Thus, the main aim of the present study is to evaluate reliability measures of a computer system by introducing the concept of priority of hardware repair over software upgradation. The system operates with one more hardware in cold standby. The failure times of hardware and software are independent random variables which follow negative exponential distribution. The repair facility (called server) attends the faults immediately which occur during operation of the system. Repair of the hardware is done at failure while software undergoes for up-gradation. The hardware repair and software up-gradation done by the server are perfect. The distributions of hardware repair and software up-gradation times are taken as arbitrary with different probability density functions. The system model has been analyzed using semiMarkov process and regenerative point technique. The trends of some important measures of system effectiveness including mean time to system failure (MTSF), availability and profit function have been observed for arbitrary values of the parameters. The profit of the present model has also been compared with that of the model Malik and Munday (2014).

\section{NOTATIONS}

\begin{tabular}{|c|c|c|}
\hline $\mathrm{E}$ & : & Set of regenerative states \\
\hline $\bar{E}$ & $:$ & Set of non-regenerative states \\
\hline $\mathrm{O}$ & $:$ & Computer system is operative \\
\hline Hcs & $:$ & Hardware is in cold standby \\
\hline $\mathrm{a} / \mathrm{b}$ & $:$ & $\begin{array}{l}\text { Probability that the system } \\
\text { has hardware / software failure }\end{array}$ \\
\hline$\lambda_{1} / \lambda_{2}$ & $:$ & $\begin{array}{l}\text { Hardware/Software failure } \\
\text { rate }\end{array}$ \\
\hline FUr /HFWr & $:$ & $\begin{array}{l}\text { The hardware is failed and } \\
\text { under repair/waiting for repair }\end{array}$ \\
\hline FUg/SFWUg & $:$ & $\begin{array}{l}\text { The software is failed and } \\
\text { under/waiting up-gradation }\end{array}$ \\
\hline IFUR/HFWR & $:$ & $\begin{array}{l}\text { The hardware is failed and } \\
\text { continuously under repair } \\
\text { waiting for repair from } \\
\text { previous state }\end{array}$ \\
\hline
\end{tabular}

SFUG/SFWUG : $\quad$ The software is failed and

continuously under up-gradation /waiting for up- gradation from previous state

\begin{tabular}{|c|c|c|}
\hline $\mathrm{g}(\mathrm{t}) / \mathrm{G}(\mathrm{t})$ & : & $\begin{array}{l}\text { pdf/cdf of hardware repair } \\
\text { time }\end{array}$ \\
\hline $\mathrm{f}(\mathrm{t}) / \mathrm{F}(\mathrm{t})$ & : & $\begin{array}{l}\text { pdf/cdf of software up- } \\
\text { gradation time }\end{array}$ \\
\hline $\mathrm{q}_{\mathrm{ij}}(\mathrm{t}) / \mathrm{Q}_{\mathrm{ij}}(\mathrm{t})$ & : & $\begin{array}{l}\text { pdf / cdf of first passage time } \\
\text { from regenerative state Si to }\end{array}$ \\
\hline
\end{tabular}


a regenerative state $S j$ or to a

failed state $\mathrm{Sj}$ without visiting

any other regenerative state in $(0, \mathrm{t}]$

\begin{tabular}{|c|c|c|}
\hline $\mathrm{q}_{\mathrm{ij} . \mathrm{k}}(\mathrm{t}) / \mathrm{Q}_{\mathrm{ij} . \mathrm{k}}(\mathrm{t})$ & $:$ & $\begin{array}{l}\text { pdf/cdf of direct transition } \\
\text { time from regenerative state } \mathrm{Si} \\
\text { to a regenerative state } \mathrm{Sj} \text { or to a } \\
\text { failed state } \mathrm{Sj} \text { visiting state } S_{\mathrm{k}} \\
\text { once in }(0, \mathrm{t}]\end{array}$ \\
\hline $\mathrm{M}_{\mathrm{i}}(\mathrm{t})$ & $:$ & $\begin{array}{l}\text { Probability that the system up } \\
\text { initially in state } S_{i} \in E \text { is up at } \\
\text { time } t \text { without visiting to any } \\
\text { regenerative state }\end{array}$ \\
\hline$W_{i}(t)$ & $:$ & $\begin{array}{l}\text { Probability that the server is } \\
\text { busy in the state } S_{i} \text { up to time ' } t \text { ' } \\
\text { without making any transition to } \\
\text { any other regenerative state or } \\
\text { returning to the same state via } \\
\text { one or more non-regenerative } \\
\text { states. }\end{array}$ \\
\hline
\end{tabular}

$\mu_{\mathrm{i}}$

The mean sojourn time in

state $S_{i}$ which is given by

$$
\begin{gathered}
\mu_{i}=E(T) \\
=\int_{0}^{\infty} P(T>t) d t=\sum_{j} m_{i j},
\end{gathered}
$$

where $T$ denotes the time to system failure.

$m_{i j}$

Contribution to mean sojourn

time $\left(\mu_{\mathrm{i}}\right)$ in state $\mathrm{S}_{\mathrm{i}}$ when system transits directly to state $S_{j}$ so that

$\mu_{i}=\sum_{j} m_{i j}$ and

$\mathrm{m}_{\mathrm{ij}}=\int_{0}^{\infty} t d Q_{i j}(t)=-q_{i j}^{*^{\prime}}(0)$

\& / C $\quad: \quad$ Symbol for Laplace-Stieltjes

convolution/Laplace

convolution

*/** $\quad: \quad$ Symbol for Laplace

Transformation (LT)/Laplace Stieltjes Transformation (LST)

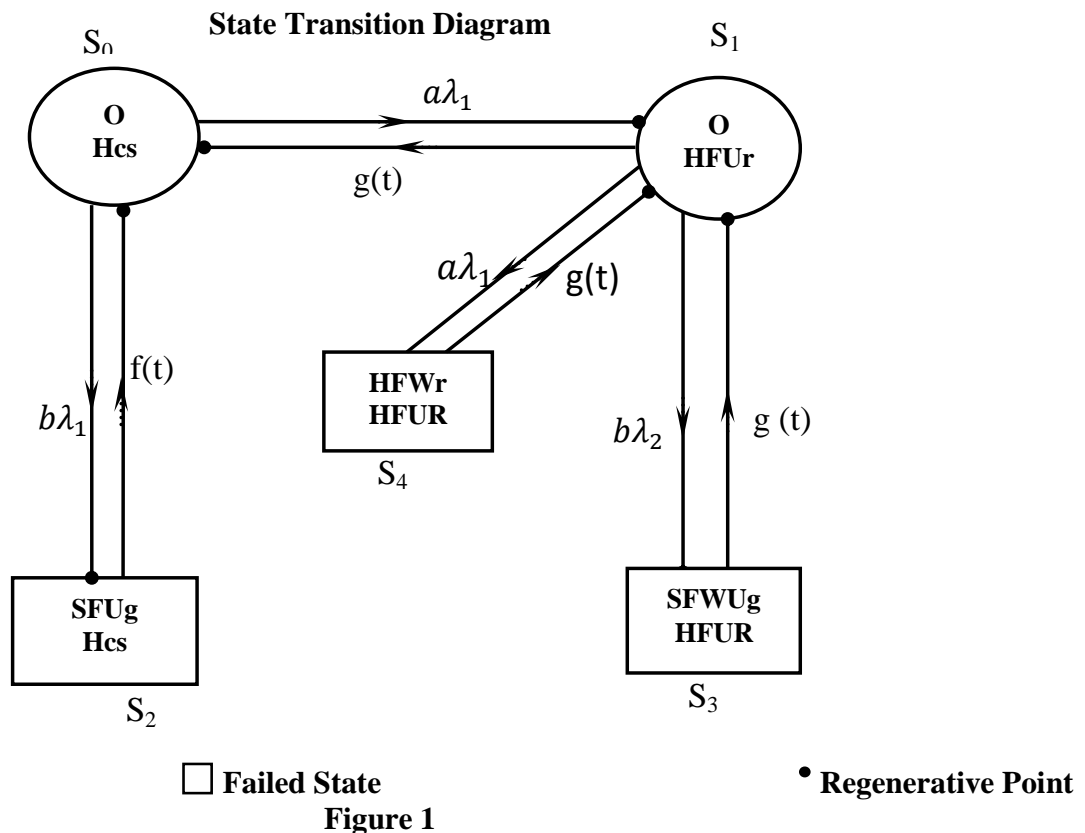

Up-State

Figure 1

\section{TRANSITION PROBABILITIES AND MEAN SOJOURN TIMES}

Simple probabilistic considerations yield the following expressions for the non-zero elements.

$$
\begin{aligned}
& p_{i j}=Q_{i j}(\infty)=\int_{0}^{\infty} q_{i j}(t) d t \\
& p_{01}=\frac{a \lambda_{1}}{a \lambda_{1}+b \lambda_{2}}, \quad p_{02}=\frac{b \lambda_{2}}{a \lambda_{1}+b \lambda_{2}}, \\
& p_{10}=g^{*}\left(a \lambda_{1}+b \lambda_{2}\right), p_{13}=\frac{b \lambda_{2}}{a \lambda_{1}+b \lambda_{2}}\left\{1-g^{*}\left(a \lambda_{1}+b \lambda_{2}\right)\right\}
\end{aligned}
$$

$$
\begin{aligned}
& p_{14}=\frac{a \lambda_{1}}{a \lambda_{1}+b \lambda_{2}}\left\{1-g^{*}\left(a \lambda_{1}+b \lambda_{2}\right)\right\}, \\
& p_{20}=f^{*}(0), p_{31}=p_{41}=g^{*}(0)
\end{aligned}
$$

For $g(t)=\alpha e^{-\alpha t}$ and $f(t)=\theta e^{-\theta t}$

But, $f^{*}(0)=g^{*}(0)=1$ and $a+b=1$

we have

$p_{11.3}=\frac{b \lambda_{2}}{a \lambda_{1}+b \lambda_{2}+\alpha} \quad, \quad p_{11.4}=\frac{a \lambda_{1}}{a \lambda_{1}+b \lambda_{2}+\alpha}$ 
It can be easily verified that

$$
\begin{aligned}
& p_{01}+p_{02}=p_{10}+p_{13}+p_{14}=p_{20}=p_{31} \\
& =p_{41}=p_{10}+p_{11.3}+p_{11.4}=1
\end{aligned}
$$

The mean sojourn times $\left(\mu_{i}\right)$ is the state $S_{i}$ are

$$
\begin{aligned}
& \mu_{0}=\frac{1}{a \lambda_{1}+b \lambda_{2}} \quad \mu_{1}=\frac{1}{a \lambda_{1}+b \lambda_{2}+\alpha} \quad \mu_{2}=\frac{1}{\theta} \\
& \mu_{3}=\mu_{4}=\frac{1}{\alpha} \quad \mu_{1}^{\prime}=\frac{1}{\alpha}
\end{aligned}
$$

Also

$\mu_{0}=m_{01}+m_{02}$

$\mu_{1}=m_{10}+m_{13}+m_{14}$

$\mu_{2}=m_{20} \quad \mu_{3}=m_{31} \quad \mu_{4}=m_{41}$

And $\mu_{1}^{\prime}=m_{10}+m_{11.3}+m_{11.4}$

\section{RELIABILITY AND MEAN TIME TO SYSTEM FAILURE (MTSF)}

Let $\phi_{i}(t)$ be the cdf of first passage time from regenerative state $S_{i}$ to a failed state. Regarding the failed state as absorbing state, we have the following recursive relations for $\phi_{i}(t)$,

$\phi_{0}(t)=Q_{01}(t) \& \phi_{1}(t)+Q_{02}(t)$

$\phi_{1}(t)=Q_{10}(t) \& \phi_{0}(t)+Q_{13}(t)+Q_{14}(t)$

Taking LST of above relations (1) and solving for $\phi_{0}^{* *}(s)$

We have

$R^{*}(s)=\frac{1-\phi_{0}^{* *}(s)}{s}$

The reliability of the system model can be obtained by taking Laplace inverse transform of the above equation. The mean time to system failure (MTSF) is given by

$M T S F=\lim _{s \rightarrow 0} \frac{1-\phi_{0}^{* *}(s)}{s}=\frac{N_{1}}{D_{1}}$

where $N_{1}=p_{01} \mu_{1}+\mu_{0}$ and $D_{1}=1-p_{01} p_{10}$

\section{STEADY STATE AVAILABILITY}

Let $A_{i}(t)$ be the probability that the system is in up-state at an instant' $t$ ' given that the system entered regenerative state $S_{i}$ at $t=0$. The recursive relations for $A_{i}(t)$ are given as:

$$
\begin{aligned}
A_{0}(t)= & M_{0}(t)+q_{01}(t) \odot A_{1}(t)+q_{02}(t) \odot A_{2}(t) \\
A_{1}(t)= & M_{1}(t)+q_{10}(t) \odot A_{0}(t) \\
& +\left\{q_{11.3}(t)+q_{11.4}(t)\right\} \odot A_{1}(t) \\
A_{2}(t)= & q_{20}(t) \Subset A_{0}(t)
\end{aligned}
$$

where

$$
M_{0}(t)=e^{-\left(a \lambda_{1}+b \lambda_{2}\right) t} \text { and } \mathrm{M}_{1}(\mathrm{t})=e^{-\left(a \lambda_{1}+b \lambda_{2}\right) t} \overline{G(t)}
$$

Taking LT of relations (4) and solving for $A_{0}^{*}(s)$, the steady state availability is given by

$A_{0}(\infty)=\lim _{s \rightarrow 0} s A_{0}^{*}(s)=\frac{N_{2}}{D_{2}}$

where $N_{2}=p_{10} \mu_{0}+p_{01} \mu_{1}$ and

$D_{2}=p_{10} \mu_{0}+p_{01} \mu_{1}^{\prime}\left(+\mu_{3}^{\prime} p_{13}\right)+p_{10} p_{02} \mu_{2}$

\section{BUSY PERIOD OF THE SERVER}

\subsection{Due to Hardware Repair}

Let $B_{i}^{H}(t)$ be the probability that the server is busy in repairing the unit due to hardware failure at an instant ' $t$ ' given that the system entered state $S_{i}$ at $t=0$. The recursive relations for $B_{i}^{H}(t)$ are as follows:

$$
\begin{aligned}
B_{0}^{H}(t)= & q_{01}(t) \odot B_{1}^{H}(t)+q_{02}(t) \subseteq B_{2}^{H}(t) \\
B_{1}^{H}(t)= & W_{1}^{H}(t)+q_{10}(t) \subseteq B_{0}^{H}(t) \\
& +\left\{q_{11.3}(t)+q_{11.4}(t)\right\} \odot B_{1}^{H}(t) \\
B_{2}^{H}(t)= & q_{20}(t) \odot B_{0}^{H}(t)
\end{aligned}
$$

where

$$
\begin{aligned}
W_{1}^{H}(t)= & e^{-\left(a \lambda_{1}+b \lambda_{2}\right) t} \overline{G(t)}+\left(a \lambda_{1} e^{-\left(a \lambda_{1}+b \lambda_{2}\right) t}(1) \overline{G(t)}\right. \\
& +\left(b \lambda_{2} e^{-\left(a \lambda_{1}+b \lambda_{2}\right) t}(1) \overline{G(t)}\right.
\end{aligned}
$$

\subsection{Due to Software Up-gradation}

Let $B_{i}^{S}(t)$ be the probability that the server is busy in upgrading the unit due to software failure at an instant ' $t$ ' given that the system entered state $S_{i}$ at $t=0$. The recursive relations for $B_{i}^{S}(t)$ are as follows:

$$
\begin{aligned}
& B_{0}^{S}(t)=q_{01}(t) \oplus B_{1}^{S}(t)+q_{02}(t) \Subset B_{2}^{S}(t) \\
& B_{1}^{S}(t)=q_{10}(t) \odot B_{0}^{S}(t)+\left\{q_{11.3}(t)+q_{11.4}(t)\right\} \subseteq B_{1}^{S}(t) \\
& B_{2}^{S}(t)=W_{2}^{S}(t)+q_{20}(t) \subset B_{0}^{S}(t)
\end{aligned}
$$

where $W_{2}^{S}(t)=\overline{F(t)}$

Taking LT of relations (7) \& (8), solving for $B_{0}^{H^{*}}(t)$ and $B_{0}^{S^{*}}(t)$. The time for which server is busy due to repairs and up-gradations respectively are given by

$B_{0}^{H}(t)=\lim _{s \rightarrow 0} s B_{0}^{H^{*}}(t)=\frac{N_{3}^{H}}{D_{2}}$
$B_{0}^{S}(t)=\lim _{s \rightarrow 0} s B_{0}^{S^{*}}(t)=\frac{N_{3}^{S}}{D_{2}}$

where

$N_{3}^{H}=p_{01} W_{1}^{H^{*}}(0), N_{3}^{S}=p_{10} p_{02} W_{2}^{S^{*}}$

$N_{3}^{R p}=p_{01} p_{13} W_{3}^{R p^{*}}(0)$ and $D_{2}$ is already mentioned.

\section{EXPECTED NUMBER OF}

\section{HARDWARE REPAIRS}

Let $N H R_{i}(t)$ be the expected number of hardware repairs by the server in $(0, t]$ given that the system entered the regenerative state $S_{i}$ at $t=0$. The recursive relations for $N H R_{i}(t)$ are given as:

$$
\begin{aligned}
N H R_{0}(t)= & Q_{01}(t) \&\left(1+N H R_{1}(t)\right) \\
& +Q_{02}(t) \& N H R_{2}(t) \\
N H R_{1}(t)= & Q_{10}(t) \& N H R_{0}(t) \\
& +\left(Q_{11.4}(t)+Q_{11.49}(t)\right) \& N H R_{1}(t) \\
N H R_{2}(t)= & Q_{20}(t) \& N H R_{0}(t)
\end{aligned}
$$

Taking LST of relations (12) and solving for $N H R_{0}^{* *}(s)$. The expected number of hardware repair is given by

$N H R_{0}=\lim _{s \rightarrow 0} s N H R_{0}^{* *}(s)=\frac{N_{4}}{D_{2}}$ 
Where, $N_{4}=p_{01} p_{10}$ and $D_{2}$ is already mentioned.

\section{EXPECTED NUMBER OF SOFTWARE UP-GRADATIONS}

Let $N S U_{i}(t)$ be the expected number of software upgradations in $(0, t]$ given that the system entered the regenerative state $S_{i}$ at $t=0$. The recursive relations for $N S U_{i}(t)$ are given as follows:

$$
\begin{aligned}
N S U_{0}(t)= & Q_{01}(t) \& N S U_{1}(t) \\
& +Q_{02}(t) \&\left(1+N S U_{2}(t)\right) \\
N S U_{1}(t)= & Q_{10}(t) \& N S U_{0}(t) \\
& +\left(Q_{11.3}(t)+Q_{11.4}(t)\right) \& N S U_{1}(t) \\
N S U_{2}(t)= & Q_{20}(t) \& N S U_{0}(t)
\end{aligned}
$$

Taking LST of relations (15) and solving for $\operatorname{NSU}_{0}^{* *}(s)$. The expected numbers of software up-gradation are given by

$$
N S U_{0}(\infty)=\lim _{s \rightarrow 0} s N S U_{0}^{* *}(s)=\frac{N_{5}}{D_{2}}
$$

Where

$N_{5}=p_{10} p_{02}$ and $D_{2}$ is already mentioned .

\section{COST-BENEFIT ANALYSIS}

The profit incurred to the system model in steady state can be obtained as:

$P=K_{0} A_{0}-K_{1} B_{0}^{H}-K_{2} B_{0}^{S}-K_{3} N H R_{0}-K_{4} N S U_{0}$

where

$K_{0}=$ Revenue per unit up-time of the system

$K_{1}=$ Cost per unit time for which server is busy due to hardware repair

$K_{2}=$ Cost per unit time for which server is busy due to software up-gradation

$K_{3}=$ Cost per unit repair of the failed hardware

$K_{4}=$ Cost per unit up-gradation of the failed software and $A_{0}, B_{0}^{H}, B_{0}^{S}, N H R_{0}, N S U_{0}$ are already defined.

\section{PARTICULAR CASES}

Suppose $g(t)=\alpha e^{-\alpha t}$ and $f(t)=\theta e^{-\theta t}$

We can obtain the following results:

$\operatorname{MTSF}\left(T_{0}\right)=\frac{N_{1}}{D_{1}}$

$\operatorname{Availability}\left(A_{0}\right)=\frac{N_{2}}{D_{2}}$

Busy period due to hardware failure $\left(B_{0}^{H}\right)=\frac{N_{3}^{H}}{D_{2}}$

Busy period due to software failure $\left(B_{0}^{S}\right)=\frac{N_{3}^{S}}{D_{2}}$
Expected number of hardware repairs $\left(N H R_{0}\right)=\frac{N_{4}}{D_{2}}$

Expected number of software upgradations $\left(N S U_{0}\right)$

$$
=\frac{N_{5}}{D_{2}}
$$

Where

$$
\begin{aligned}
& N_{1}=\frac{2 a \lambda_{1}+b \lambda_{2}+\alpha}{\left(a \lambda_{1}+b \lambda_{2}\right)\left(a \lambda_{1}+b \lambda_{2}+\alpha\right)} \\
& D_{1}=\frac{\left(a \lambda_{1}+b \lambda_{2}\right)\left(a \lambda_{1}+b \lambda_{2}+\alpha\right)-\alpha a \lambda_{1}}{\left(a \lambda_{1}+b \lambda_{2}\right)\left(a \lambda_{1}+b \lambda_{2}+\alpha\right)} \\
& N_{2}=\frac{\alpha+a \lambda_{1}}{\left(a \lambda_{1}+b \lambda_{2}\right)\left(a \lambda_{1}+b \lambda_{2}+\alpha\right)} \\
& D_{2}=\frac{\alpha^{2}\left(b \lambda_{2}+\theta\right)+\theta a \lambda_{1}\left(a \lambda_{1}+b \lambda_{2}+\alpha\right)}{\alpha \theta\left(a \lambda_{1}+b \lambda_{2}\right)\left(a \lambda_{1}+b \lambda_{2}+\alpha\right)} \\
& N_{3}^{H}=\frac{a \lambda_{1}}{\left(a \lambda_{1}+b \lambda_{2}\right)\left(a \lambda_{1}+b \lambda_{2}+\alpha\right)} \\
& N_{3}^{S}=\frac{\alpha b \lambda_{2}}{\theta\left(a \lambda_{1}+b \lambda_{2}\right)\left(a \lambda_{1}+b \lambda_{2}+\alpha\right)} \\
& N_{4}=\frac{\alpha a \lambda_{1}}{\left(a \lambda_{1}+b \lambda_{2}\right)\left(a \lambda_{1}+b \lambda_{2}+\alpha\right)} \\
& N_{5}=\frac{\alpha b \lambda_{2}}{\left(a \lambda_{1}+b \lambda_{2}\right)\left(a \lambda_{1}+b \lambda_{2}+\alpha\right)}
\end{aligned}
$$

\section{CONCLUSION}

The results for a particular case are obtained to depict the behaviour of some important reliability measures such as mean time to system failure (MTSF), availability and profit function as shown respectively in figures 2,3 , and 4 . It is analyzed that these measures go on decreasing with the increase of failure rates $\left(\lambda_{1}\right.$ and $\left.\lambda_{2}\right)$. However, they keep on increasing with the increase of hardware repair rate $(\alpha)$ and software up-gradation rate $(\theta)$ provided system has more chances of hardware failure than that of software failure $(\mathrm{a}>\mathrm{b})$. It is interesting to note that system becomes more profitable by interchanging the values of $a$ and $b(a<b)$. Hence, study reveals that a computer system with hardware redundancy in cold standby can be made more profitable by giving priority to hardware repair over software up-gradation. The comparison of profit of the system model is also shown in figure 5 .

\section{ACKNOWLEDGEMENT}

The authors are grateful to the University Grants Commission (UGC), New Delhi, India for providing financial Assistance to carry out this research work under Rajiv Gandhi National Fellowship for SC Candidate.

\section{REFERENCES}

[1]. Anand, Jyoti and Malik, S.C. (2012): Analysis of a Computer System with Arbitrary Distributions for H/W and S/W Replacement Time and Priority to Repair Activities of $\mathrm{H} / \mathrm{W}$ over Replacement of the $\mathrm{S} / \mathrm{W}$, International Journal of Systems Assurance Engineering and Management, Vol.3 (3), pp. 230-236.

[2]. Kumar, Ashish; Anand, Jyoti and Malik, S.C. (2013): Stochastic Modeling of a Computer System with Priority to Up-gradation of Software over Hardware Repair 
Activities. International Journal of Agricultural and Statistical Sciences, Vol. 9(1), pp. 117-126.

[3]. Malik, S.C. and Anand, Jyoti (2010): Reliability and economic analysis of a computer system with independent hardware and software failures. Bulletin of Pure and Applied Sciences, Vol. 29 E (01), pp.141-153.

[4]. Malik, S.C. and Munday, V.J. (2014): Stochastic Modeling of a Computer System with Hardware Redundancy, International Journal of Computer applications, Vol. 89 (7), pp. 26-30.
[5]. Malik, S.C. (2013): Reliability Modeling of a computer System with Preventive Maintenance and Priority Subject to Maximum Operation and Repair Times. International Journal of System Assurance Engineering and Management, Vol. 4 (1), pp. 94-100.

[6]. Welke, S. R., Labib, S. W. and Ahmed, A. M. (1995): Reliability Modeling of Hardware/ Software System, IEEE Transactions on Reliability, Vol.44, No.3, pp.413418

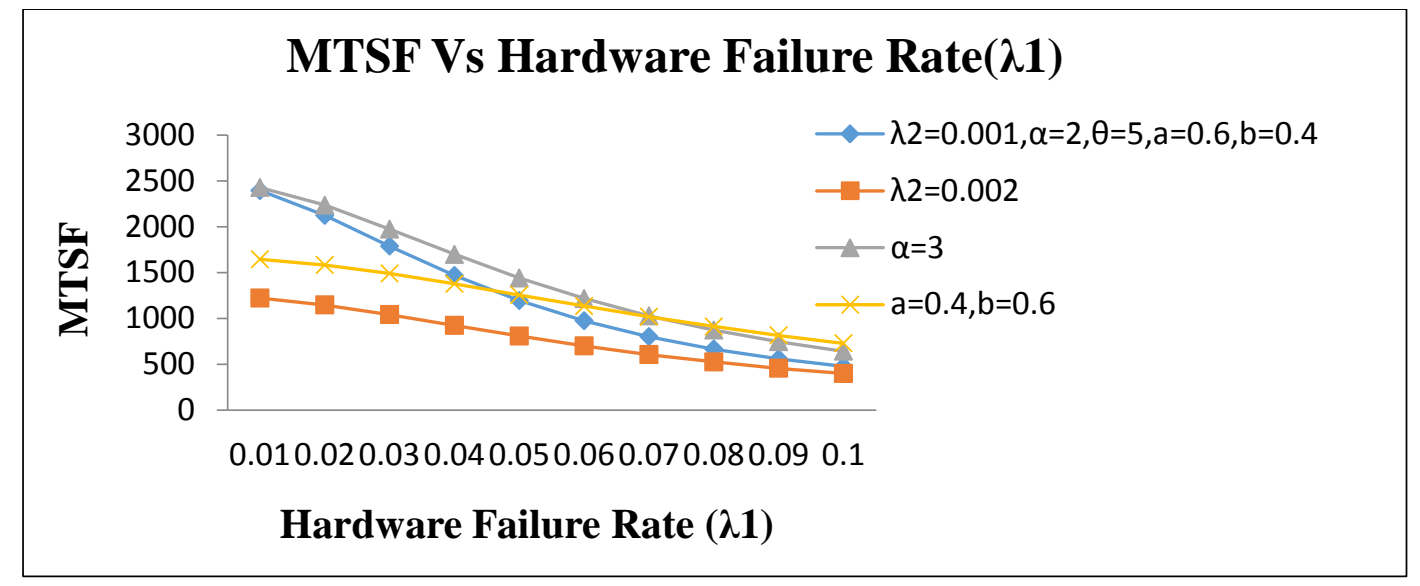

Fig:2

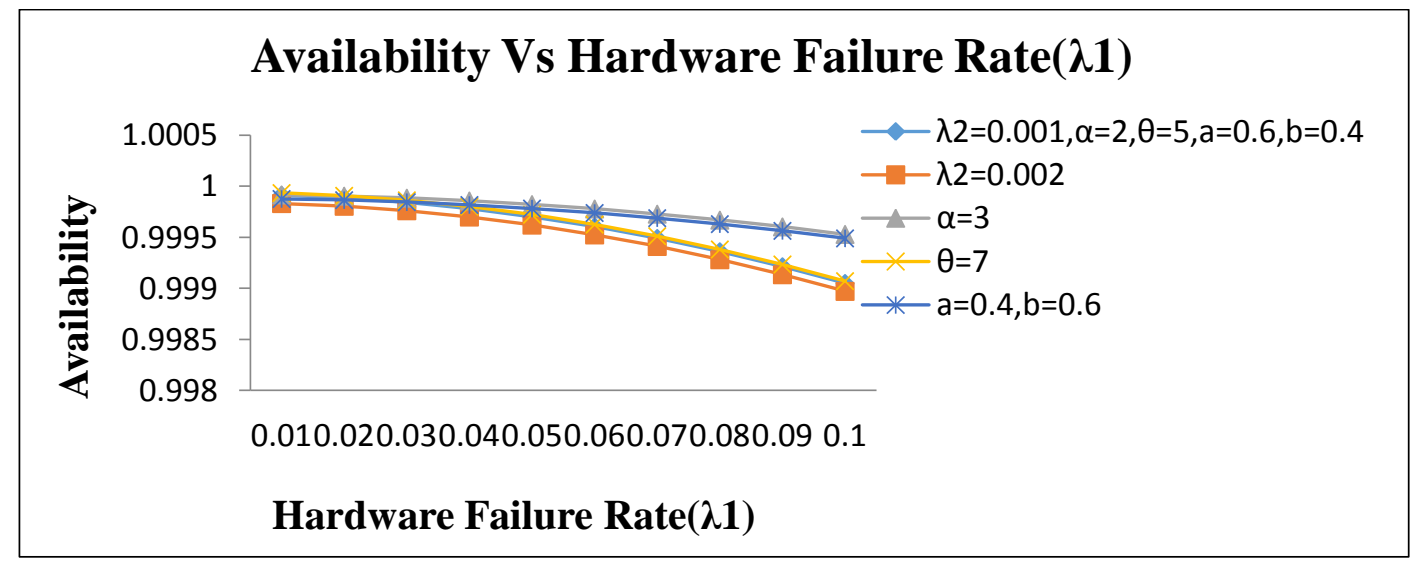

Fig:3

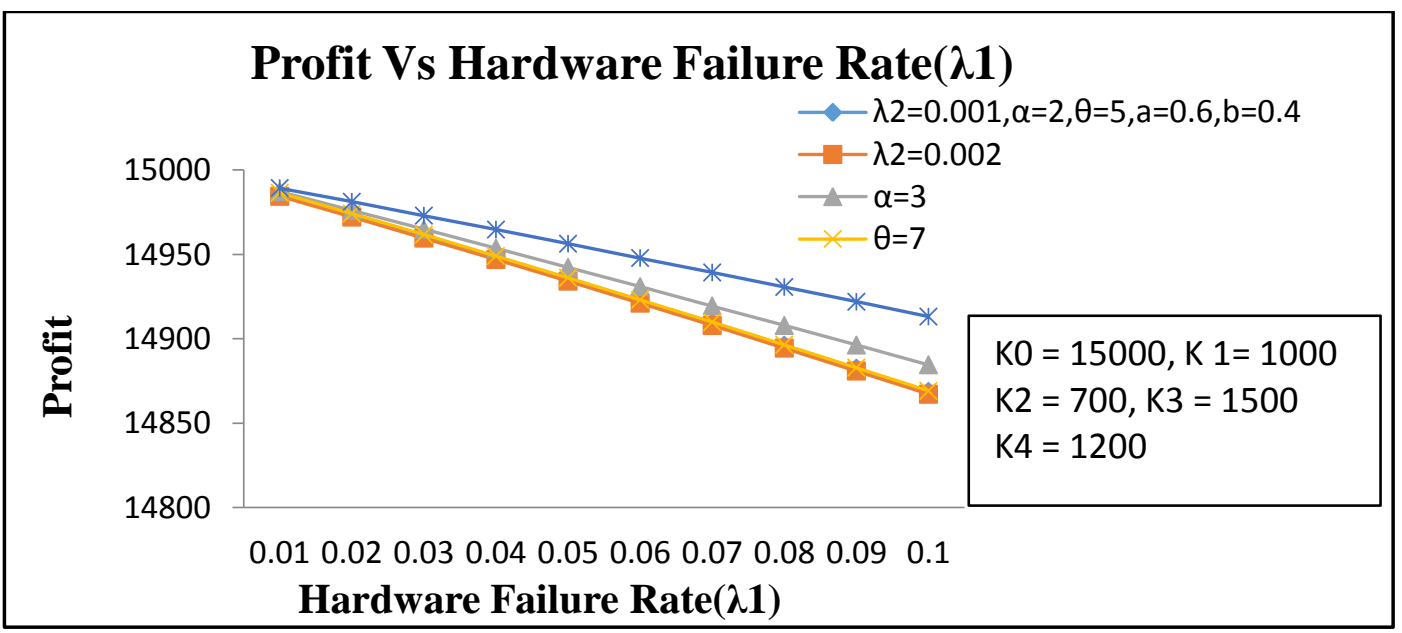

Fig:4 
P - Profit of the present model and P1 - Profit of the model Malik and Munday (2014) Graph of Profit Difference (P - P1)

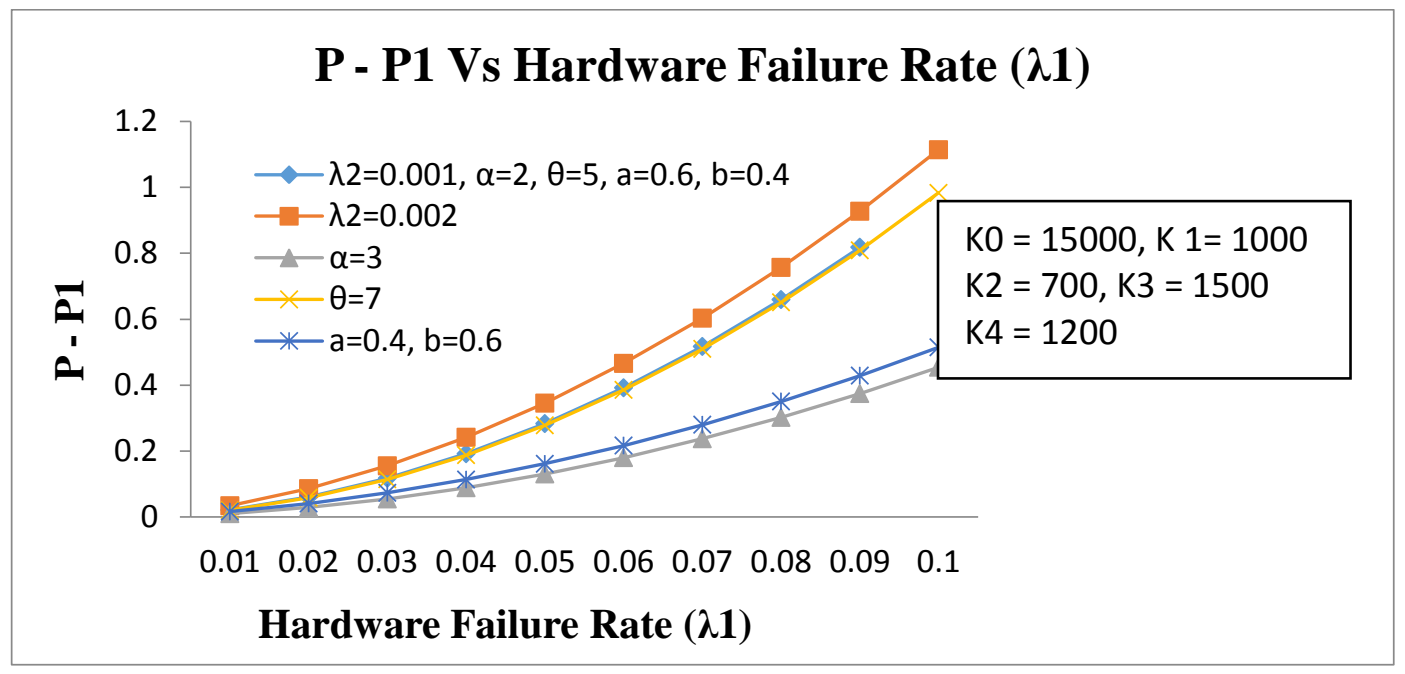

Fig. 5 